\title{
Audio Message Transmitter Secured Through Elliptical Curve Cryptosystem
}

\author{
Artan Luma ${ }^{*}$, Besnik Selimi , Lirim Ameti
}

\author{
Accepted $15^{\text {th }}$ August 2014
}

\begin{abstract}
Securing a communication is always a challenge for participants in it. A lot of applications available in the market claim to enable secure audio communication, but not always show the details of the technology used behind to encrypt the data. It is important for end users to understand the techniques used for encrypting the data, in order to trust it. Elliptic curve cryptography, an approach to public key cryptography, is now widely used in cryptographic systems. Hence, in this paper we propose using elliptic curve cryptography to secure the transmission of voice messages through the network. The system that is proposed and implemented enables the encryption of the voice message, its transmission through the network and its decryption at the other end.
\end{abstract}

Keywords: privacy, cryptography, elliptic curves, voice message (.wav).

\section{Introduction}

The success of the business processes today is closely linked to the efficiency of the privacy of communication between the parties. Our aim is to secure the transmission of voice messages. As mentioned in the abstract part, there are plenty of applications in the market who claim they enable a secure audio communication, without telling about the relying technology, and this makes us to suspect about their level of security.

Although known algorithms as RSA, are in wide use today, they are not suitable for use when dealing with large amounts of data upon which needs to be applied an efficient cryptographic system, always taking into account along with the privacy, the performance too.

Voice messages compared to text messages [1], contain a large amount of data and this is why the aforementioned algorithms are not efficient because of their performance.

Our proposed system addresses this issue. The elliptic curve cryptography is more than appropriate for achieving the goal. Compared to RSA algorithm, the efficiency of elliptic curve cryptography is stated as follows: "Safety of elliptical curves is based on elliptic curve discrete logarithm problem (ECDLP) which enables ECC to reach the same level of security with RSA for smaller keys and greater computation efficiency. ECC-160 provides security compared with RSA-1024 and ECC-224 provides security compared with RSA-2048 [2]". This fact is sufficient to build our cryptographic system based on elliptical curves, which is the main purpose this paper.

The rest of this paper is organized as follows: section 2 describes elliptical curve operations, section 3 describes the encryption and decryption with elliptical curves, section 4 describes the audio format (.wav) in a way as will be used in our system, section 5 explains the system setup and section 6 concludes this paper.

\footnotetext{
${ }^{I}$ Contemporary Sciences and Technologies, South East European University, Campus, 1200, Tetovo/Macedonia

* Corresponding Author: Email: a.luma@seeu.edu.mk

\# This paper has been presented at the International Conference on Advanced Technology\&Sciences (ICAT'14) held in Antalya (Turkey), August 12-15, 2014.
}

\section{Elliptical Curve Operations}

Elliptic curve operations which are of interest of our paper are: point addition, point subtraction, point doubling and point multiplication. For these operations to be faster, more accurate and more efficient, the elliptical curve cryptography is defined over two finite fields:

--Prime field $F_{p}$, where $\mathrm{p}$ is a prime,

--Binary field $F_{2}^{m}$, where $\mathrm{m}$ is a positive integer.

We use the prime field $F_{p}$, where as a case study we consider the following elliptical curve:

$y^{2} \equiv x^{3}+x+1(\bmod 277)$, or $E_{277}(1,1)$

Elliptic curve operations as point addition, point subtraction, point doubling and point multiplication are defined as following:

\subsection{Point addition}

Consider two distinct points $\mathrm{J}$ and $\mathrm{K}$ such that $\mathrm{J}=\left(\mathrm{x}_{\mathrm{j}}, \mathrm{y}_{\mathrm{j}}\right)$ and $\mathrm{K}=\left(\mathrm{x}_{\mathrm{k}}, \mathrm{y}_{\mathrm{k}}\right)$.

Let $\mathrm{L}=\mathrm{J}+\mathrm{K}$, where $\mathrm{L}=\left(\mathrm{x}_{\mathrm{L}}, \mathrm{y}_{\mathrm{L}}\right)$, then:

$\mathrm{x}_{\mathrm{L}} \equiv \mathrm{s}^{2}-\mathrm{x}_{\mathrm{j}}-\mathrm{x}_{\mathrm{k}}(\bmod \mathrm{p})$

$\mathrm{y}_{\mathrm{L}} \equiv-\mathrm{y}_{\mathrm{j}}+\mathrm{s}\left(\mathrm{x}_{\mathrm{J}}-\mathrm{x}_{\mathrm{L}}\right)(\bmod \mathrm{p})$

$\mathrm{s} \equiv\left(\mathrm{y}_{\mathrm{L}}-\mathrm{y}_{\mathrm{K}}\right) /\left(\mathrm{x}_{\mathrm{J}}-\mathrm{x}_{\mathrm{K}}\right)(\bmod \mathrm{p})$

$\mathrm{s}$ is the slope of the line through $\mathrm{J}$ and $\mathrm{K}$.

If $K=-J$, i.e. $K \equiv\left(x_{J},-y_{J}\right)(\bmod p)$ then $J+K=0$, where 0 is the point at infinity.

If $\mathrm{K}=\mathrm{J}$ then $\mathrm{J}+\mathrm{K}=2 \cdot \mathrm{J}$ then point doubling operations are used. Also:

$\mathrm{J}+\mathrm{K}=\mathrm{K}+\mathrm{J}$

Let $\mathrm{J}=(1,130)$ and $\mathrm{K}=(4,30)$ then the point $\mathrm{L}\left(\mathrm{x}_{\mathrm{L}}, \mathrm{y}_{\mathrm{L}}\right)$ can be calculated as:

First we calculate : 


$$
\begin{aligned}
s & \equiv \frac{30-130}{4-1}(\bmod 277) \\
\mathrm{s} & \equiv \frac{-100}{3}(\bmod 277) \\
\mathrm{s} & \equiv-100 \cdot \frac{1}{3}(\bmod 277) \\
\mathrm{s} & \equiv(-100) \cdot(-92)(\bmod 277) \\
\mathrm{s} & \equiv 9200(\bmod 277) \\
\mathrm{s} & \equiv 59(\bmod 277) \\
& \text { then } \\
\mathrm{x}_{\mathrm{L}} & \equiv\left(59^{2}-1-4\right)(\bmod 277) \\
\mathrm{x}_{\mathrm{L}} & \equiv(3481-5)(\bmod 277) \\
\mathrm{x}_{\mathrm{L}} & \equiv 3476(\bmod 277) \\
\mathrm{x}_{\mathrm{L}} & \equiv 152(\bmod 277) \\
\text { and } & \\
\mathrm{y}_{\mathrm{L}} & \equiv(59 \cdot(1-152)-130)(\bmod 277) \\
\mathrm{y}_{\mathrm{L}} & \equiv(59 \cdot(-151)-130)(\bmod 277) \\
\mathrm{y}_{\mathrm{L}} & \equiv(-8909-130)(\bmod 277) \\
\mathrm{y}_{\mathrm{L}} & \equiv-9039(\bmod 277) \\
\mathrm{y}_{\mathrm{L}} & \equiv 102(\bmod 277) \\
&
\end{aligned}
$$

Hence the result of point addition of $(1,130)$ and $(4,30)$ for the elliptic group $\mathrm{E}_{277}(1,1)$ is $(152,102)$.

\subsection{Point subtraction}

Consider two distinct points $\mathrm{J}$ and $\mathrm{K}$ such that $\mathrm{J}=\left(\mathrm{x}_{\mathrm{J}}, \mathrm{y}_{\mathrm{J}}\right)$ and $\mathrm{K}=\left(\mathrm{x}_{\mathrm{K}}, \mathrm{y}_{\mathrm{K}}\right)$, then $\mathrm{J}-\mathrm{K}=\mathrm{J}+(-\mathrm{K})$, where $-\mathrm{K} \equiv\left(\mathrm{x}_{\mathrm{K}},-\mathrm{y}_{\mathrm{K}}\right)(\bmod \mathrm{p})$.

Let $\mathrm{J}=(1,130)$ and $\mathrm{K}=(4,30)$, then $-\mathrm{K} \equiv(4,-30) \equiv$ $(4,247)(\bmod 277)$ and

$\mathrm{L}=\mathrm{J}-\mathrm{K}=\mathrm{J}+(-\mathrm{K})$

$\mathrm{L}=(1,130)+(4,247)=(131,63)$

Hence with the subtraction of $J=(1,130)$ and $K=(4,30)$, i.e. $\mathrm{L}=\mathrm{J}-\mathrm{K}$, as a result is gained the point $\mathrm{L}=(131,63)$ which also lies in our elliptical curve.

\subsection{Point doubling}

Consider a point $\mathrm{J}$ such that $\mathrm{J}=\left(\mathrm{x}_{\mathrm{J}}, \mathrm{y}_{\mathrm{J}}\right)$, where $\mathrm{y}_{\mathrm{J}} \neq 0$. Let $\mathrm{L}=2 \cdot \mathrm{J}$, where $\mathrm{L}=\left(\mathrm{x}_{\mathrm{L}}, \mathrm{y}_{\mathrm{L}}\right)$, then:

$\mathrm{x}_{\mathrm{L}} \equiv \mathrm{s}^{2}-2 \cdot \mathrm{x}_{\mathrm{J}}(\bmod \mathrm{p})$

$\mathrm{y}_{\mathrm{L}} \equiv-\mathrm{y}_{\mathrm{J}}+\mathrm{s} \cdot\left(\mathrm{x}_{\mathrm{J}}-\mathrm{x}_{\mathrm{L}}\right)(\bmod \mathrm{p})$

$s \equiv\left(3 \cdot x_{J}^{2}+a\right) /\left(2 \cdot y_{J}\right)(\bmod p)$

$\mathrm{S}$ is the tangent at point $\mathrm{J}$ and $\mathrm{a}$ is one of the parameters chosen with the elliptic curve.

If $\mathrm{y}_{\mathrm{J}}=0$, then $2 \cdot \mathrm{J}=0$, where 0 is the point at infinity. Let $J=(1,130)$ and $K=(4,30)$ then the point $L(x L, y L)$ can be calculated as:

Let $\mathrm{J}=(1,130)$ and we calculate the point $\mathrm{L}$, i.e. $\mathrm{L}=2 \cdot \mathrm{J}$. Firstly we calculate s: $s \equiv\left(\frac{3 \cdot 1^{2}+1}{2 \cdot 130}\right)(\bmod 277)$

$\mathrm{s} \equiv\left(\frac{4}{260}\right)(\bmod 277)$

$\mathrm{s} \equiv\left(4 \cdot \frac{1}{260}\right)(\bmod 277)$

$\mathrm{s} \equiv(4 \cdot 114)(\bmod 277)$

$s \equiv 456(\bmod 277)$

$\mathrm{s} \equiv 179(\bmod 277)$

then

$\mathrm{x}_{\mathrm{L}} \equiv\left(179^{2}-2 \cdot 1\right)(\bmod 277)$

$\mathrm{x}_{\mathrm{L}} \equiv(32041-2)(\bmod 277)$

$\mathrm{x}_{\mathrm{L}} \equiv 32039(\bmod 277)$

$\mathrm{x}_{\mathrm{L}} \equiv 184(\bmod 277)$

and

$\mathrm{y}_{\mathrm{L}} \equiv\left(\mathrm{s} \cdot\left(\mathrm{x}_{\mathrm{J}}-\mathrm{x}_{\mathrm{L}}\right)-\mathrm{y}_{\mathrm{J}}\right)(\bmod 277)$

$\mathrm{y}_{\mathrm{L}} \equiv(179 \cdot(1-184)-130)(\bmod 277)$

$\mathrm{y}_{\mathrm{L}} \equiv(179 \cdot(-183)-130)(\bmod 277)$

$\mathrm{y}_{\mathrm{L}} \equiv(-32757-130)(\bmod 277)$

$\mathrm{y}_{\mathrm{L}} \equiv-32887(\bmod 277)$

$\mathrm{y}_{\mathrm{L}} \equiv 76(\bmod 277)$

Hence the result of doubling of the point $(1,130)$ for the group $\mathrm{E}_{277}(1,1)$ is the point $(184,76)[3]$.

\subsection{Point multiplication}

We calculate point multiplication by combining point addition and point multiplication, an algorithm called as double-and-add which functions like following:

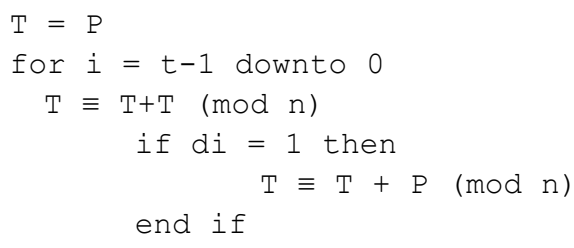

where $\mathrm{P}$ is a point in the elliptical curve, $\mathrm{T}$ is the variable where the result is stored, $t$ is the binary width of the scalar which multiplies the point and $d_{i}$ is the bit with the index $i$ [4].

Consider the point $\mathrm{P}(1,130)$ that lies in the curve. Let us take a scalar $\mathrm{d}=47$, i.e. $\mathrm{d}=(101111)_{2}$ and $\mathrm{d}_{\mathrm{i}}=[1,1,1,1,0,1]$, then $\mathrm{T}=\mathrm{d} \cdot \mathrm{P}$, i.e. $\mathrm{T}=47 \cdot(1,130)$. Based on the aforementioned algorithm are following the calculations:

$\mathrm{T}=(1,130)$

$\mathrm{i}=6-1=5, \quad \mathrm{~T}=((1,130)+(1,130))(\bmod 277)$

$\mathrm{d}_{5}=1, \quad \mathrm{~T}=((184,76)+(1,130))(\bmod 277)$

$\mathrm{i}=5-1=4, \mathrm{~T}=((67,3)+(67,3))(\bmod 277)$

$\mathrm{d}_{4}=0, \quad /$ 


$$
\begin{aligned}
& \mathrm{i}=4-1=3, \quad \mathrm{~T}=((103,73)+(103,73))(\bmod 277) \\
& \mathrm{d}_{3}=1, \quad \mathrm{~T}=((228,100)+(1,130))(\bmod 277) \\
& \mathrm{i}=3-1=2, \quad \mathrm{~T}=((137,10)+(137,10))(\bmod 277) \\
& \mathrm{d}_{2}=1, \quad \mathrm{~T}=((103,204)+(1,130))(\bmod 277) \\
& \mathrm{i}=2-1=1, \quad \mathrm{~T}=((60,1)+(60,1))(\bmod 277) \\
& \mathrm{d}_{1}=1, \quad \mathrm{~T}=((158,97)+(1,130))(\bmod 277) \\
& \mathrm{i}=1-1=0, \quad \mathrm{~T}=((192,46)+(192,46))(\bmod 277) \\
& \mathrm{d}_{0}=1, \quad \mathrm{~T}=((227,102)+(1,130))(\bmod 277) \\
& \mathrm{T}=(46,106)
\end{aligned}
$$

Hence the multiplication of point $\mathrm{P}=(1,130)$ with the scalar $\mathrm{d}=47$, in the elliptical group $\mathrm{E}_{277}(1,1)$ gives as a result the point $\mathrm{T}=\mathrm{d} \cdot \mathrm{P}=(46,106)$.

\section{ECC Encryption / Decryption}

Elliptic curve cryptography can be used to encrypt a plaintext message, say $M$, into ciphertext. The plaintext message $M$ is encoded into a point $\mathrm{P}_{\mathrm{M}}$ from the finite set of points in the elliptic group, $\mathrm{E}_{\mathrm{P}}(\mathrm{a}, \mathrm{b})$. The first step consists in choosing a generator point, $G \in E_{P}(a, b)$ such that the smaller value of $n$ for which $\mathrm{n} \cdot \mathrm{G}=0$ is a very large prime number. The elliptic group $\mathrm{E}_{\mathrm{P}}(\mathrm{a}, \mathrm{b})$ and the generator point $\mathrm{G}$ are made public.

Each user select a private key, $\mathrm{n}_{\mathrm{A}}<n$ and compute the public key $P_{A}=n_{A} \cdot G$. To encrypt the message point $P_{M}$ for $B, A$ choses a random integer $k$ and compute the ciphertext pair of points $P_{C}$ using B's public key $\mathrm{P}_{\mathrm{B}}$ :

$$
\mathrm{P}_{\mathrm{C}}=\left[(\mathrm{k} \cdot \mathrm{G}),\left(\mathrm{P}_{\mathrm{M}}+\mathrm{k} \cdot \mathrm{P}_{\mathrm{B}}\right)\right]
$$

After receiving the ciphertext pair of points $P_{C}$, B multiplies the first point, $(\mathrm{k} \cdot \mathrm{G})$ with his private key $\mathrm{n}_{\mathrm{B}}$ and then adds the result to the second point in the ciphertext pair of points, $\left(\mathrm{P}_{\mathrm{M}}+\mathrm{k} \cdot \mathrm{P}_{\mathrm{B}}\right)$ :

$$
\left(\mathrm{P}_{\mathrm{M}}+\mathrm{k} \cdot \mathrm{P}_{\mathrm{B}}\right)-\left(\mathrm{n}_{\mathrm{B}} \cdot \mathrm{k} \cdot \mathrm{G}\right)=
$$

$=\left(P_{M}+k \cdot n_{B} \cdot G\right)-\left(n_{B} \cdot k \cdot G\right)=P_{M}$

which is the plaintext point, corresponding to the plaintext message M. Only $B$, knowing the private key $n_{B}$, can remove $n_{B} \cdot(k \cdot G)$ from the second point of the ciphertext pair of point, i.e. $\left(\mathrm{P}_{\mathrm{M}}+\mathrm{k} \cdot \mathrm{P}_{\mathrm{B}}\right)$, and hence retrieve the plaintext information $\mathrm{P}_{\mathrm{M}}$ [5].

Consider our elliptic curve:

$\mathrm{y}^{2} \equiv \mathrm{x}^{3}+\mathrm{x}+1(\bmod 277)$

That is $a=1, b=1, p=277$. The elliptic curve group generated by the above elliptic curve is $E_{p}(a, b)=E_{277}(1,1)$.

Let the generator point $G=(0,276)$, then multiplies $\mathrm{k} \cdot \mathrm{G}$ of the generator point $\mathrm{G}$ are (for $1 \leq \mathrm{k} \leq 277$ ):

$$
\begin{aligned}
& G=(0,276) \quad 2 G=(208,105) \quad 3 G=(72,220) \\
& 4 G=(274,91) \quad 5 G=(174,74) \quad 6 G=(84,3) \\
& 7 G=(117,35) \quad 8 G=(47,157) \quad 9 G=(146,241) \\
& 10 G=(122,201) \quad \ldots \ldots \ldots \quad 274 G=(121,180) \\
& 275 G=(258,34) \quad 276 G=(149,188) \quad 277 G=(175,175)
\end{aligned}
$$

If $A$ wants to send to $B$ the message $M$ which is encoded as the plaintext point $\mathrm{P}_{\mathrm{M}}=(18,158) \in \mathrm{E}_{277}(1,1)$. A must use $\mathrm{B}$ 's public key to encrypt it. Suppose that B's secret key is $n_{B}=85$, then B's public key will be:

$P_{B}=n_{B} \cdot G=85 \cdot(0,276)$

$\mathrm{P}_{\mathrm{B}}=(237,15)$
A selects a random number $\mathrm{k}=113$ and uses B's public key $P_{B}=(237,15)$ to encrypt the message point into the ciphertext pair of points:

$$
\begin{aligned}
& P_{C}=\left[(k \cdot G),\left(P_{M}+k \cdot P_{B}\right)\right] \\
& P_{C}=[113 \cdot(0,276),(18,158)+113 \cdot(237,15)] \\
& P_{C}=[(260,67),(18,158)+(253,130)] \\
& P_{C}=[(260,67),(68,178)]
\end{aligned}
$$

Upon receiving the ciphertext pair of points $P_{C}=[(260,67),(68,178)], B$ uses his private key $n_{B}=85$, to compute the plaintext point $\mathrm{P}_{\mathrm{M}}$ as follows:

$$
\begin{aligned}
& \left(P_{M}+k \cdot P_{B}\right)-\left[n_{B} \cdot(k \cdot G)\right]=(68,178)-[85 \cdot(260,67)] \\
& \left(P_{M}+k \cdot P_{B}\right)-\left[n_{B} \cdot(k \cdot G)\right]=(68,178)-(253,130) \\
& \left(P_{M}+k \cdot P_{B}\right)-\left[n_{B} \cdot(k \cdot G)\right]=(68,178)+(253,-130) \\
& \text { because }-P=(x P,-y P) \\
& \left(P_{M}+k \cdot P_{B}\right)-\left[n_{B}(k \cdot G)\right]=(68,178)+(253,147) \\
& \text { because }-130 \equiv 147(\bmod 277) \\
& \left(P_{M}+k \cdot P_{B}\right)-\left[n_{B}(k \cdot G)\right]=(18,158)
\end{aligned}
$$

and then maps the plaintext point $\mathrm{P}_{\mathrm{M}}=(18,158)$ back into the original plaintext message $\mathrm{M}$.

\section{Message Format}

Since the purpose of the whole paper is to build a cryptosystem based on elliptical curves to secure the transmission of voice messages, in this section we will examine the integral structure of one particular file format (WAVE) upon which we will apply encryption and decryption.

The WAVE file format is a subset of Microsoft's RIFF specification for the storage of multimedia files. A RIFF file starts out with a file header followed by a sequence of data chunks. A WAVE file is often just a RIFF file with a single "WAVE" chunk which consists of two sub-chunks -- a "fmt" chunk specifying the data format and a "data" chunk containing the actual sample data [6]. 


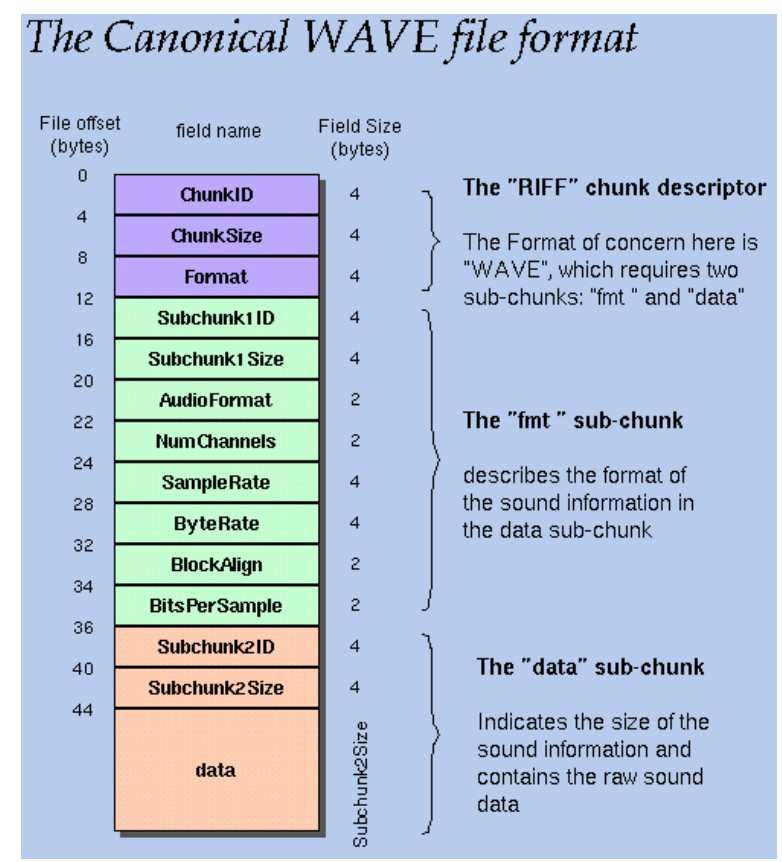

Figure 1. The structure of .wav file format.

The screen above clearly shows that the actual data is stored after the 44-th byte and for illustrative reasons in our implementation will encrypt only the part of actual data which is stored from the 45-th byte till the end of the file, thus allowing the file to be playable but producing meaningless noise (encrypted voice).

\section{Implementation}

Software solution for the introduced system in this paper is implemented in $\mathrm{C \#}$ language, with the interface as in the following figure:

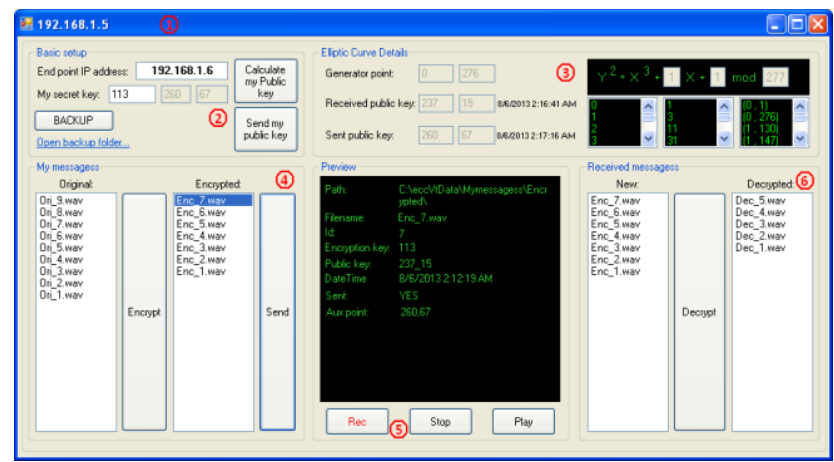

Figure 2. System interface.

\subsection{Encryption of voice message}

The voice message is read and its bytes are stored in an array 1sOriTB. The array is then split into two other arrays where the first array lsOriH contains the first 44 bytes which represent the header bytes, while the second array lsOriD contains the remaining bytes which represent the actual data of the voice.

lsOriH won't be encrypted in order to enable playing the file. The bytes from lsOriD will be mapped into corresponding points of the elliptic curve which are stored in the arrays lsMapX and lsMapY.

The encryption algorithm described in section 3 is applied upon the stored points in the arrays lsMapX and lsMapY. The encrypted points are stored in the arrays 1sEX and lsEY. The bytes from $1 \mathrm{sEX}$ and $1 \mathrm{sEY}$ are mapped back into the corresponding points and stored into the array lsED.

lsOriH and lsED are merged into lsEncTB and the content is written in a .wav file. The file represents the encrypted message which plays a meaningless noise, and this way could be securely transmitted through the network.

\subsection{Decryption of voice message}

The encrypted voice message is read and its bytes are stored in the array lsEncTB. The array then is split into two other arrays where the first array lsOriH contains the first 44 bytes which represent the header bytes, while the second array $1 \mathrm{sEncD}$ contains the following bytes which represent the actual data of the encrypted voice.

lsOriH won't be decrypted since it represents the original header. The bytes from $1 \mathrm{sEncD}$ are mapped into corresponding points of the elliptic curve which are stored in the arrays lsMapX and lsMapY.

The decryption algorithm described in section 3 is applied upon the stored points in the arrays lsMapX and lsMapY. The decrypted points are stored in the arrays $1 \mathrm{SOX}$ and lsOY.

The bytes from 1sOX and 1sOY are mapped back into the corresponding points and stored into the array lsOD.

$1 \mathrm{sOriH}$ and 1sOD are merged into lsOriTB and the content is written in a .wav file. The file represents the decrypted message which plays the original message, hence the goal of this paper is achieved.

\subsection{Transmission of voice message}

Transmission of messages is done using sockets [7]. The main path of the system operation is illustrated as follows:

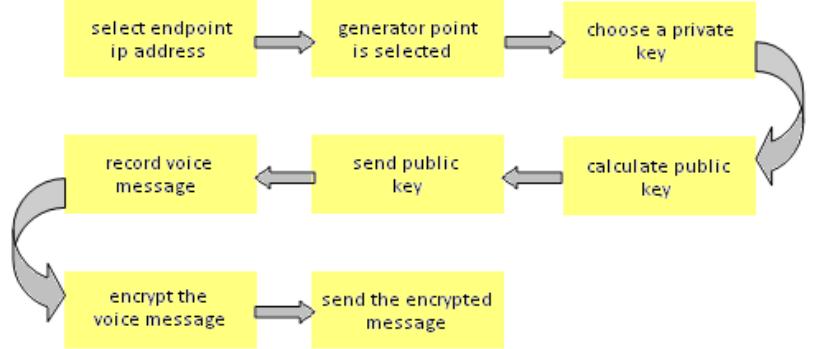

Figure 3. System main path operation.

To make the system more stable, during transmission of the voice message, encryption public data related to the message are also transmitted, which gives flexibility in changing and exchanging keys.

\subsection{Data organization}

The data that our system operates with are organized in files and folders. Also there is a folder for data backup. The organization of the data is better depicted by the following figure:

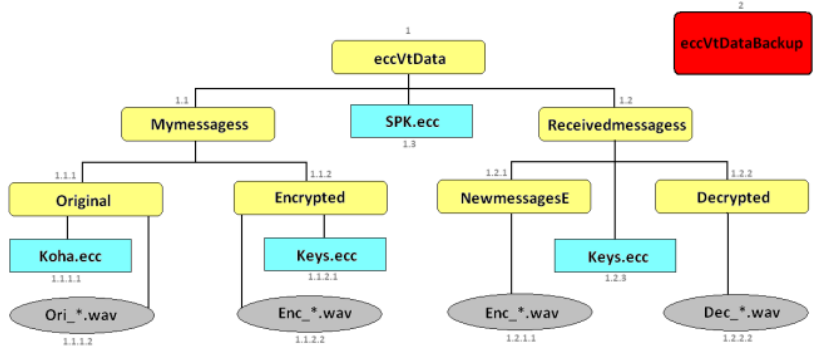

Figure 4. Data organization.

Files of type ecc are files in which are stored voice message details such as: recording time, encryption and decryption keys, transmission time etc. 


\section{Conclusion and Future Work}

In this paper we have proposed and implemented a cryptographic system based on elliptic curves, adapted to provide secure audio communication between communicating parties.

Using a similar approach, one can use elliptic curves for encryption of other types of data like image, video, text. The advantage of elliptic curves relies in the fact that using a smallerlength key results in a stronger encryption compared to RSA encryption.

Considering the prospect of elliptic curves in terms of cryptosystems, there remain to work on optimizing the provided solution and adapt it for an implementation which will enable secure real time mobile communication.

\section{References}

[1] Shoewu, O. and S.O. Olatinwo. 2013. "Securing Text Messages using Elliptic Curve Cryptography Orthogonal Frequency Division Multiplexing".

[2] Nils Gura, Arun Patel, Arvinderpal Wander, Comparing Elliptic Curve Cryptography and RSA on 8-bit CPUs, 2004.

[3] Fuwen Liu, A Tutorial on Elliptic Curve Cryptography (ECC),https://www-rnks.informatik.tucottbus.de/content/unrestricted/staff/lfw/A\%20tutorial\%20o f\%20elliptic\%20curve\%20cryptography.pdf [15.12.2013].

[4] Christof Paar and Jan Pelzi, Understanding Cryptography, http://wiki.crypto.rub.de/Buch/download/Understanding_Cr yptography_Chptr_9---ECC.pdf [15.12.2013].

[5] Design of Secure Computer Systems CSI4138/CEG4394, Dr Jean-Yves Chouinard, 2002.

[6] WAVE PCM soundfile format. https://ccrma.stanford.edu/courses/422/projects/WaveForm at/ [15.12.2013].

[7] Socket Class, http://msdn.microsoft.com/enus/library/system.net.sockets.socket(v=vs.110).aspx [15.12.2013] 